Table of Contents (TOC)

Concise Synthesis oflsoquinoline via the Ugi and Heck Reactions

Zheng Xiang, Tuoping Luo, Kui Lu, Jiayue Cui, Xiaomeng Shi, Reza Fathi* Jiahua Chen* and Zhen Yang*

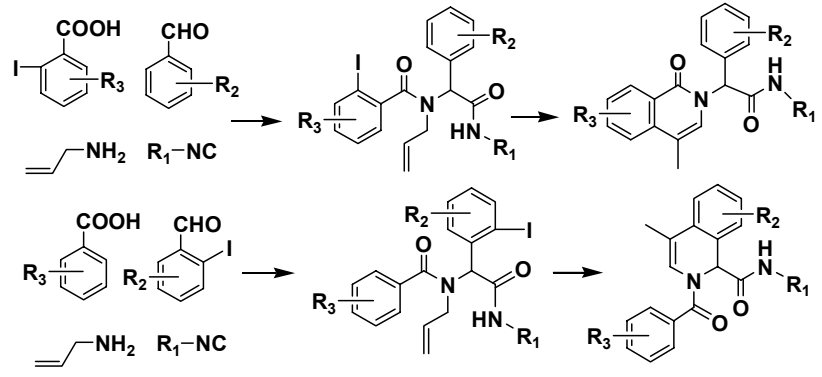




\title{
Concise Synthesis of Isoquinoline via the Ugi and Heck
}

\section{Reactions}

\author{
Zheng Xiang, ${ }^{\dagger}$ Tuoping Luo, ${ }^{\dagger}$ Kui Lu, ${ }^{\dagger}$ Jiayue Cui,${ }^{\dagger}$ \\ Xiaomeng Shi, ${ }^{\dagger}$ Reza Fathi, ${ }^{*}{ }^{\star}$ Jiahua Chen ${ }^{*, \dagger}$ and Zhen Yang ${ }^{*, \dagger, \dagger}$
}

Key Laboratory of Bioorganic Chemistry and Molecular Engineering of Ministry of Education, College of Chemistry, Beijing, 100871 and Laboratory of Chemical Genetics, Shenzhen Graduate School of Peking University, The University Town, Shenzhen, 518055, China, and VivoQuest, Inc. 711 Executive Blvd, Valley Cottage, NY, 10989, USA.

zyang@chem.pku.edu.cn

\section{Supporting Information}

\section{General Information}

$\mathrm{Pd}(\mathrm{OAc})_{2}$ and $\mathrm{PCy}_{3}$ were purchased from Aldrich and used without further purification. DMA and $\mathrm{N}$-methyldicyclohexylamine were purified according to "Purification of Laboratory Chemicals ( $3^{\text {rd }}$ edition)" by D. D. Perrin and W. L. F. Armarego just before using. The boiling point of petroleum ether is between $60-90^{\circ} \mathrm{C}$. Silica gel (200-300 mesh) for purification was purchased from Qing Dao Hai Yang Chemical Industry Co. of China. ${ }^{1} \mathrm{H}-\mathrm{NMR}$ and ${ }^{13} \mathrm{C}-\mathrm{NMR}$ were recorded at $300 \mathrm{MHz}$ and $75 \mathrm{MHz}$ with Varian Mercury 300 spectrometer or at $400 \mathrm{MHz}$ and 100.6 $\mathrm{MHz}$ with Brucker ARX400 spectrometer. Mass spectrometric data were obtained using ZAB-HS mass spectrometer.

All the Heck reactions were carried out in glassware that was flame-dried and cooled under nitrogen.

\section{General Procedure}

\section{Ugi reaction}

To a solution of allylamine $(1.0 \mathrm{mmol})$ in $\mathrm{MeOH}(1.0 \mathrm{~mL})$ was added aldehyde $(1.0 \mathrm{mmol})$, and the reaction mixture was stirred at room temperature for $10 \mathrm{~min}$. Acid $(1.0 \mathrm{mmol})$ was added to the reaction mixture. After stirring for another $5 \mathrm{~min}$ isocyanide $(1.0 \mathrm{mmol})$ was added. The reaction mixture was stirred overnight. Solvent was removed under reduced pressure and the 
residue was purified by flash chromatography (EtOAc/ $\mathrm{CH}_{2} \mathrm{Cl}_{2} /$ petroleum ether) to give the corresponding Ugi product.

\section{Heck reaction}

$\mathrm{Pd}(\mathrm{OAc})_{2}(5.6 \mathrm{mg}, 0.025 \mathrm{mmol}), \mathrm{Pcy}_{3}(14.0 \mathrm{mg}, 0.05 \mathrm{mmol})$ and Ugi product $(0.5 \mathrm{mmol})$ were added to a dry Schlenk tube under nitrogen. DMA $(5.0 \mathrm{~mL})$ and $N$-methyldicyclohexylamine $(0.42$ $\mathrm{mL}, 2.0 \mathrm{mmol}$ ) were added under nitrogen. The reaction mixture was stirred at $100{ }^{\circ} \mathrm{C}$ or $60^{\circ} \mathrm{C}$ for 4 to 24 hours. Solvent was removed under reduced pressure and the residue was purified by flash chromatography $\left(\mathrm{EtOAc} / \mathrm{CH}_{2} \mathrm{Cl}_{2}\right.$ /petroleum ether $=1: 1: 5$ to $\left.1: 1: 1\right)$ to give the corresponding Heck product.

\section{Compound 1ab}<smiles>Cc1cn(C(C(=O)NC(C)(C)C)c2ccc([N+](=O)[O-])cc2)c(=O)c2ccccc12</smiles>

$\mathrm{Pd}(\mathrm{OAc})_{2}(5.6 \mathrm{mg}, 0.025 \mathrm{mmol}), \mathrm{Pcy}_{3}(14.0 \mathrm{mg}, 0.05 \mathrm{mmol})$ and $1 \mathrm{aa}(260.7 \mathrm{mg}, 0.5 \mathrm{mmol})$ were added to a flame-dried Schlenk tube under nitrogen. DMA $(5.0 \mathrm{~mL})$ and $N$-methyldicyclohexylamine $(0.42 \mathrm{~mL}, 2.0 \mathrm{mmol})$ were added under nitrogen. The reaction mixture was stirred at $100{ }^{\circ} \mathrm{C}$ overnight. Solvent was removed under reduced pressure and the residue was purified by flash chromatography $\left(\mathrm{EtOAc} / \mathrm{CH}_{2} \mathrm{Cl}_{2} /\right.$ petroleum ether $\left.=1: 1: 5\right)$ to give $181.1 \mathrm{mg}$ of the corresponding Heck product $1 \mathrm{ab}\left(92 \%\right.$ yield). ${ }^{1} \mathrm{H}$ NMR $\left(300 \mathrm{MHz}, \mathrm{CDCl}_{3}\right): 1.40$ (s, 9H), $2.22(\mathrm{~s}, 3 \mathrm{H}), 6.20(\mathrm{br} \mathrm{s}, 1 \mathrm{H}), 6.89(\mathrm{~s}, 1 \mathrm{H}), 6.94(\mathrm{~s}, 1 \mathrm{H}), 7.52-7.63(\mathrm{~m}, 4 \mathrm{H}), 7.71-7.74(\mathrm{~m}, 1 \mathrm{H})$, $8.24(\mathrm{~d}, J=9.0 \mathrm{~Hz}, 2 \mathrm{H}), 8.47(\mathrm{~d}, J=8.1 \mathrm{~Hz}, 1 \mathrm{H}) ;{ }^{13} \mathrm{C} \mathrm{NMR}\left(75 \mathrm{MHz}, \mathrm{CDCl}_{3}\right)$ : $\delta 15.6,28.6,52.1$, 59.5, 112.7, 123.3, 124.0, 124.9, 126.8, 127.0, 128.2, 129.5, 132.7, 137.3, 143.5, 147.7, 162.1, 166.7; LRMS (EI): $393\left(\mathrm{M}^{+}\right)$; HRMS (EI): calcd forC ${ }_{22} \mathrm{H}_{23} \mathrm{~N}_{3} \mathrm{O}_{4}\left(\mathrm{M}^{+}\right) 393.1689$, found 393.1681.

\section{Compound 2ab}<smiles>Cc1cn(C(C(=O)NC(C)(C)C)c2ccc(Cl)cc2)c(=O)c2ccccc12</smiles>

$\mathrm{Pd}(\mathrm{OAc})_{2}(5.6 \mathrm{mg}, 0.025 \mathrm{mmol}), \mathrm{Pcy}_{3}(14.0 \mathrm{mg}, 0.05 \mathrm{mmol})$ and $2 \mathbf{a a}(255.4 \mathrm{mg}, 0.5 \mathrm{mmol})$ were added to a flame-dried Schlenk tube under nitrogen. DMA (5.0 mL) and $\mathrm{N}$-methyldicyclohexylamine $(0.42 \mathrm{~mL}, 2.0 \mathrm{mmol})$ were added under nitrogen. The reaction mixture was stirred at $100{ }^{\circ} \mathrm{C}$ overnight. Solvent was removed under reduced pressure and the residue was purified by flash chromatography $\left(\mathrm{EtOAc} / \mathrm{CH}_{2} \mathrm{Cl}_{2} /\right.$ petroleum ether $\left.=1: 1: 5\right)$ to give $181.4 \mathrm{mg}$ of the corresponding Heck product $2 \mathbf{a b}$ (95\% yield). ${ }^{1} \mathrm{H}$ NMR $\left(300 \mathrm{MHz}, \mathrm{CDCl}_{3}\right): 1.37$ (s, 9H), $2.20(\mathrm{~s}, 3 \mathrm{H}), 6.39(\mathrm{~s}, 1 \mathrm{H}), 6.90(\mathrm{~s}, 1 \mathrm{H}), 6.94(\mathrm{~s}, 1 \mathrm{H}), 7.36(\mathrm{~s}, 4 \mathrm{H}), 7.47-7.58(\mathrm{~m}, 2 \mathrm{H})$, 
7.67-7.72 (m, 1H), $8.41(\mathrm{~d}, J=8.1 \mathrm{~Hz}, 1 \mathrm{H}) ;{ }^{13} \mathrm{C} \mathrm{NMR}\left(75 \mathrm{MHz}, \mathrm{CDCl}_{3}\right): \delta 15.6,28.6,51.9,59.6$, 111.9, 123.1, 125.1, 126.5, 127.4, 128.3, 129.2, 130.2, 132.4, 134.5, 134.7, 137.3, 162.1, 167.4; LRMS (EI): $382\left(\mathrm{M}^{+}\right)$; HRMS (EI): calcd forC ${ }_{22} \mathrm{H}_{23} \mathrm{~N}_{2} \mathrm{O}_{2}{ }^{35} \mathrm{Cl}\left(\mathrm{M}^{+}\right) 382.1448$, found 382.1451 .

\section{Compound 3ab}<smiles>Cc1cn(C(C(=O)NC(C)(C)C)c2ccccc2[N+](=O)[O-])c(=O)c2ccccc12</smiles>

$\mathrm{Pd}(\mathrm{OAc})_{2}(5.6 \mathrm{mg}, 0.025 \mathrm{mmol}), \mathrm{Pcy}_{3}(14.0 \mathrm{mg}, 0.05 \mathrm{mmol})$ and 3aa $(260.7 \mathrm{mg}, 0.5 \mathrm{mmol})$ were added to a flame-dried Schlenk tube under nitrogen. DMA $(5.0 \mathrm{~mL})$ and $N$-methyldicyclohexylamine $(0.42 \mathrm{~mL}, 2.0 \mathrm{mmol})$ were added under nitrogen. The reaction mixture was stirred at $100{ }^{\circ} \mathrm{C}$ overnight. Solvent was removed under reduced pressure and the residue was purified by flash chromatography $\left(\mathrm{EtOAc} / \mathrm{CH}_{2} \mathrm{Cl}_{2} /\right.$ petroleum ether $\left.=1: 1: 5\right)$ to give 185.0mg of the corresponding Heck product 3ab (94\% yield). ${ }^{1} \mathrm{H}$ NMR $\left(400 \mathrm{MHz}, \mathrm{CDCl}_{3}\right): 1.37$ (s, 9H), 2.29 (s, 3H), $6.14(\mathrm{~s}, 1 \mathrm{H}), 7.04(\mathrm{~s}, 1 \mathrm{H}), 7.07(\mathrm{~d}, J=8.0 \mathrm{~Hz}, 1 \mathrm{H}), 7.29(\mathrm{~s}, 1 \mathrm{H}), 7.46-7.55$ (m, $3 \mathrm{H}), 7.62-7.64(\mathrm{~m}, 1 \mathrm{H}), 7.72-7.74(\mathrm{~m}, 1 \mathrm{H}), 8.13(\mathrm{dd}, J=1.2,8.0 \mathrm{~Hz}, 1 \mathrm{H}), 8.41-8.43(\mathrm{~m}, 1 \mathrm{H}) ;{ }^{13} \mathrm{C}$ NMR $\left(75 \mathrm{MHz}, \mathrm{CDCl}_{3}\right): \quad \delta 15.6,28.4,52.3,60.1,113.3,123.3,125.4,126.2,127.0,128.5,128.6$, 128.9, 131.6, 132.7, 133.4, 137.2, 149.0, 131.8, 166.6; LRMS (EI): $393\left(\mathrm{M}^{+}\right)$; HRMS (EI): calcd for $\mathrm{C}_{22} \mathrm{H}_{23} \mathrm{~N}_{3} \mathrm{O}_{4}\left(\mathrm{M}^{+}\right)$393.1689, found 393.1683.

\section{Compound 4ab}<smiles>Cc1cn(C(C(=O)NC2CCCCC2)c2ccccc2[N+](=O)[O-])c(=O)c2ccccc12</smiles>

$\mathrm{Pd}(\mathrm{OAc})_{2}(5.6 \mathrm{mg}, 0.025 \mathrm{mmol}), \mathrm{Pcy}_{3}(14.0 \mathrm{mg}, 0.05 \mathrm{mmol})$ and $4 \mathrm{aa}(273.7 \mathrm{mg}, 0.5 \mathrm{mmol})$ were added to a flame-dried Schlenk tube under nitrogen. DMA $(5.0 \mathrm{~mL})$ and $N$-methyldicyclohexylamine $(0.42 \mathrm{~mL}, 2.0 \mathrm{mmol})$ were added under nitrogen. The reaction mixture was stirred at $100{ }^{\circ} \mathrm{C}$ overnight. Solvent was removed under reduced pressure and the residue was purified by flash chromatography $\left(\mathrm{EtOAc} / \mathrm{CH}_{2} \mathrm{Cl}_{2} /\right.$ petroleum ether $\left.=1: 1: 5\right)$ to give $195.6 \mathrm{mg}$ of the corresponding Heck product $4 \mathbf{a b}\left(93 \%\right.$ yield). ${ }^{1} \mathrm{H}$ NMR $\left(300 \mathrm{MHz}, \mathrm{CDCl}_{3}\right)$ : 1.02-1.40 (m, 5H), 1.56-1.83 (m, 4H), 1.95-2.00 (m, 1H), $2.28(\mathrm{~s}, 3 \mathrm{H}), 3.73-3.81(\mathrm{~m}, 1 \mathrm{H}), 6.09(\mathrm{~s}$, $1 \mathrm{H}), 7.02(\mathrm{~s}, 1 \mathrm{H}), 7.10(\mathrm{~d}, J=7.8 \mathrm{~Hz}, 1 \mathrm{H}), 7.35(\mathrm{~s}, 1 \mathrm{H}), 7.46-7.51(\mathrm{~m}, 3 \mathrm{H}), 7.64(\mathrm{~d}, J=8.1 \mathrm{~Hz}$, 1H), 7.73-7.78 (m, 1H), $8.12(\mathrm{dd}, J=1.8,7.8 \mathrm{~Hz}, 1 \mathrm{H}), 8.45(\mathrm{~d}, J=8.1 \mathrm{~Hz}, 1 \mathrm{H}) ;{ }^{13} \mathrm{C} \mathrm{NMR}$ $\left(75 \mathrm{MHz}, \mathrm{CDCl}_{3}\right): \quad \delta 15.6,24.7,32.5,32.7,49.2,59.5,113.4,123.3,125.4,126.3,127.0,128.5$, 128.6, 128.9, 131.4, 132.7, 133.4, 137.2, 149.0, 161.9, 166.3; LRMS (EI): 419 (M+); HRMS (EI): calcd forC ${ }_{24} \mathrm{H}_{25} \mathrm{~N}_{3} \mathrm{O}_{4}\left(\mathrm{M}^{+}\right) 419.1845$, found 419.1828 .

\section{Compound 5ab}


<smiles></smiles>

$\mathrm{Pd}(\mathrm{OAc})_{2}(5.6 \mathrm{mg}, 0.025 \mathrm{mmol}), \mathrm{Pcy}_{3}(14.0 \mathrm{mg}, 0.05 \mathrm{mmol})$ and $5 \mathrm{aa}(273.7 \mathrm{mg}, 0.5 \mathrm{mmol})$ were added to a flame-dried Schlenk tube under nitrogen. DMA (5.0 mL) and $N$-methyldicyclohexylamine $(0.42 \mathrm{~mL}, 2.0 \mathrm{mmol})$ were added under nitrogen. The reaction mixture was stirred at $100{ }^{\circ} \mathrm{C}$ overnight. Solvent was removed under reduced pressure and the residue was purified by flash chromatography $\left(\mathrm{EtOAc} / \mathrm{CH}_{2} \mathrm{Cl}_{2} /\right.$ petroleum ether $\left.=1: 1: 5\right)$ to give $193.3 \mathrm{mg}$ of the corresponding Heck product $5 \mathbf{a b}\left(92 \%\right.$ yield). ${ }^{1} \mathrm{H}$ NMR $\left(300 \mathrm{MHz}, \mathrm{CDCl}_{3}\right)$ : 1.07-1.39 (m, 5H), 1.57-1.84 (m, 3H), 1.88-2.00 (m, 1H), 2.02-2.05 (m, 1H), 2.2.28 (s, 3H), 2.23 (s, 3H), 3.83-3.88 (m, 3H), $6.78(\mathrm{~d}, J=7.8 \mathrm{~Hz}, 1 \mathrm{H}), 7.05(\mathrm{~s}, 2 \mathrm{H}), 7.50-7.62(\mathrm{~m}, 4 \mathrm{H}), 7.71-7.76(\mathrm{~m}$, $1 \mathrm{H}), 8.20(\mathrm{~d}, J=6.9 \mathrm{~Hz}, 2 \mathrm{H}), 8.40(\mathrm{~d}, J=7.5 \mathrm{~Hz}, 1 \mathrm{H}) ;{ }^{13} \mathrm{C} \mathrm{NMR}\left(75 \mathrm{MHz}, \mathrm{CDCl}_{3}\right): \delta 15.6,24.7$, $25.3,32.5,32.8,49.0,59.2,113.0,123.3,123.9,124.8,126.8,127.0,128.2,129.3,132.8,137.3$, 143.4, 147.6, 162.2, 166.4; LRMS (EI): $419\left(\mathrm{M}^{+}\right)$; HRMS (EI): calcd forC $_{24} \mathrm{H}_{25} \mathrm{~N}_{3} \mathrm{O}_{4}\left(\mathrm{M}^{+}\right)$ 419.1845, found 419.1826 .

\section{Compound 6ab}<smiles>COc1ccc(C(C(=O)NC2CCCCC2)n2cc(C)c3ccccc3c2=O)cc1</smiles>

$\mathrm{Pd}(\mathrm{OAc})_{2}(5.6 \mathrm{mg}, 0.025 \mathrm{mmol}), \mathrm{Pcy}_{3}(14.0 \mathrm{mg}, 0.05 \mathrm{mmol})$ and $6 \mathbf{6 a}(266.2 \mathrm{mg}, 0.5 \mathrm{mmol})$ were added to a flame-dried Schlenk tube under nitrogen. DMA (5.0 mL) and $N$-methyldicyclohexylamine $(0.42 \mathrm{~mL}, 2.0 \mathrm{mmol})$ were added under nitrogen. The reaction mixture was stirred at $100{ }^{\circ} \mathrm{C}$ overnight. Solvent was removed under reduced pressure and the residue was purified by flash chromatography $\left(\mathrm{EtOAc} / \mathrm{CH}_{2} \mathrm{Cl}_{2} /\right.$ petroleum ether $\left.=1: 1: 5\right)$ to give $186.5 \mathrm{mg}$ of the corresponding Heck product 6ab $\left(92 \%\right.$ yield). ${ }^{1} \mathrm{H}$ NMR $\left(300 \mathrm{MHz}, \mathrm{CDCl}_{3}\right)$ : $1.08-1.37(\mathrm{~m}, 5 \mathrm{H}), 1.55-1.66(\mathrm{~m}, 3 \mathrm{H}), 1.87-2.00(\mathrm{~m}, 2 \mathrm{H}), 3.79-3.86(\mathrm{~m}, 4 \mathrm{H}), 6.88-6.91(\mathrm{~m}, 3 \mathrm{H})$, $7.01(\mathrm{~s}, 1 \mathrm{H}), 7.35(\mathrm{~d}, J=8.7 \mathrm{~Hz}, 2 \mathrm{H}), 7.45-7.50(\mathrm{~m}, 1 \mathrm{H}), 7.55(\mathrm{~d}, J=8.1 \mathrm{~Hz}, 1 \mathrm{H}), 7.64-7.70(\mathrm{~m}$, $1 \mathrm{H}), 8.42(\mathrm{~d}, J=7.8 \mathrm{~Hz}, 1 \mathrm{H}) ;{ }^{13} \mathrm{C}$ NMR $\left(75 \mathrm{MHz}, \mathrm{CDCl}_{3}\right): \delta 15.6,24.7,25.3,32.5,32.8,48.7$, $55.2,59.7,111.5,114.2,122.9,125.2,126.2,127.6,127.8,128.3,130.2,132.2,137.3,159.5$, 162.1, 167.8; LRMS (EI): $404\left(\mathrm{M}^{+}\right)$; HRMS (EI): calcd forC $\mathrm{C}_{25} \mathrm{H}_{28} \mathrm{~N}_{2} \mathrm{O}_{3}\left(\mathrm{M}^{+}\right)$404.2100, found 404.2100 .

\section{Compound 7ab}


<smiles></smiles>

$\mathrm{Pd}(\mathrm{OAc})_{2}(5.6 \mathrm{mg}, 0.025 \mathrm{mmol}), \mathrm{Pcy}_{3}(14.0 \mathrm{mg}, 0.05 \mathrm{mmol})$ and $7 \mathbf{a a}(268.4 \mathrm{mg}, 0.5 \mathrm{mmol})$ were added to a flame-dried Schlenk tube under nitrogen. DMA (5.0 mL) and $N$-methyldicyclohexylamine $(0.42 \mathrm{~mL}, 2.0 \mathrm{mmol})$ were added under nitrogen. The reaction mixture was stirred at $100{ }^{\circ} \mathrm{C}$ overnight. Solvent was removed under reduced pressure and the residue was purified by flash chromatography $\left(\mathrm{EtOAc} / \mathrm{CH}_{2} \mathrm{Cl}_{2} /\right.$ petroleum ether $\left.=1: 1: 5\right)$ to give $196.7 \mathrm{mg}$ of the corresponding Heck product $7 \mathbf{a b}$ (96\% yield). ${ }^{1} \mathrm{H}$ NMR $\left(300 \mathrm{MHz}, \mathrm{CDCl}_{3}\right)$ : $1.07-1.41(\mathrm{~m}, 5 \mathrm{H}), 1.55-173(\mathrm{~m}, 3 \mathrm{H}), 1.81-1.84(\mathrm{~m}, 1 \mathrm{H}), 1.96-2.00(\mathrm{~m}, 1 \mathrm{H}), 2.21(\mathrm{~s}, 3 \mathrm{H})$, 3.78-3.88 (m, 1H), $6.61(\mathrm{~s}, 1 \mathrm{H}), 6.94(\mathrm{~s}, 1 \mathrm{H}), 7.02(\mathrm{~s}, 1 \mathrm{H}), 7.34(\mathrm{~s}, 4 \mathrm{H}), 7.47-7.60(\mathrm{~m}, 1 \mathrm{H}), 7.58(\mathrm{~d}$, $J=7.8 \mathrm{~Hz}, 1 \mathrm{H}), 7.68-7.73(\mathrm{~m}, 1 \mathrm{H}), 8.40(\mathrm{~d}, J=7.8 \mathrm{~Hz}, 1 \mathrm{H}) ;{ }^{13} \mathrm{C} \mathrm{NMR}\left(75 \mathrm{MHz}, \mathrm{CDCl}_{3}\right): \delta 15.5$, 24.7, 25.3, 32.5, 32.8, 48.8, 59.2, 112.1, 123.1, 124.9, 126.4, 127.4, 128.2, 129.0, 132.4, 134.3, 134.6, 137.2, 162.1, 167.1; LRMS (EI): $408\left(\mathrm{M}^{+}\right)$; HRMS (EI): calcd forC ${ }_{24} \mathrm{H}_{25} \mathrm{~N}_{2} \mathrm{O}_{2}{ }^{35} \mathrm{Cl}\left(\mathrm{M}^{+}\right)$ 408.1605 , found 408.1621 .

\section{Compound 8ab}<smiles>Cc1cn(C(C(=O)NC(C)(C)C)c2ccccc2[N+](=O)[O-])c(=O)c2c(F)cccc12</smiles>

$\mathrm{Pd}(\mathrm{OAc})_{2}(5.6 \mathrm{mg}, 0.025 \mathrm{mmol}), \mathrm{Pcy}_{3}(14.0 \mathrm{mg}, 0.05 \mathrm{mmol})$ and $8 \mathbf{a a}(269.7 \mathrm{mg}, 0.5 \mathrm{mmol})$ were added to a flame-dried Schlenk tube under nitrogen. DMA (5.0 mL) and $N$-methyldicyclohexylamine $(0.42 \mathrm{~mL}, 2.0 \mathrm{mmol})$ were added under nitrogen. The reaction mixture was stirred at $100{ }^{\circ} \mathrm{C}$ overnight. Solvent was removed under reduced pressure and the residue was purified by flash chromatography $\left(\mathrm{EtOAc} / \mathrm{CH}_{2} \mathrm{Cl}_{2} /\right.$ petroleum ether $\left.=1: 1: 5\right)$ to give $166.8 \mathrm{mg}$ of the corresponding Heck product 8ab (81\% yield). ${ }^{1} \mathrm{H}$ NMR $\left(300 \mathrm{MHz}, \mathrm{CDCl}_{3}\right): 1.34$ (s, 9H), $2.26(\mathrm{~s}, 3 \mathrm{H}), 6.22(\mathrm{~s}, 1 \mathrm{H}) 7.06(\mathrm{~s}, 1 \mathrm{H}), 7.10-7.16(\mathrm{~m}, 2 \mathrm{H}), 7.24(\mathrm{~s}, 1 \mathrm{H}), 7.38(\mathrm{~d}, J=8.1 \mathrm{~Hz}$, $1 \mathrm{H}), 7.40-7.58(\mathrm{~m}, 2 \mathrm{H}), 7.63-7.66(\mathrm{~m}, 1 \mathrm{H}), 8.13(\mathrm{dd}, J=1.5,8.1 \mathrm{~Hz}, 1 \mathrm{H}) ;{ }^{13} \mathrm{C}$ NMR $(75 \mathrm{MHz}$, $\left.\mathrm{CDCl}_{3}\right): \quad \delta 16.1,28.4,30.9,52.3,59.6,112.3,114.1,114.4,119.2,128.5,127.6,128.7,129.0$, 131.4, 133.4, 133.7, 133.8, 139.9, 149.0, 161.2, 166.6; LRMS (EI): $411\left(\mathrm{M}^{+}\right)$; HRMS (EI): calcd forC ${ }_{22} \mathrm{H}_{22} \mathrm{~N}_{3} \mathrm{O}_{4} \mathrm{~F}\left(\mathrm{M}^{+}\right)$411.1594, found 411.1603.

\section{Compound 9ab}<smiles>COc1cc(OC)c2c(C)cn(C(C(=O)NC(C)(C)C)c3ccc([N+](=O)[O-])cc3)c(=O)c2c1</smiles> 
$\mathrm{Pd}(\mathrm{OAc})_{2}(5.6 \mathrm{mg}, 0.025 \mathrm{mmol}), \mathrm{Pcy}_{3}(14.0 \mathrm{mg}, 0.05 \mathrm{mmol})$ and 9aa $(290.7 \mathrm{mg}, 0.5 \mathrm{mmol})$ were added to a flame-dried Schlenk tube under nitrogen. DMA $(5.0 \mathrm{~mL})$ and $N$-methyldicyclohexylamine $(0.42 \mathrm{~mL}, 2.0 \mathrm{mmol})$ were added under nitrogen. The reaction mixture was stirred at $100{ }^{\circ} \mathrm{C}$ overnight. Solvent was removed under reduced pressure and the residue was purified by flash chromatography $\left(\mathrm{EtOAc} / \mathrm{CH}_{2} \mathrm{Cl}_{2} /\right.$ petroleum ether $\left.=1: 1: 5\right)$ to give $170.5 \mathrm{mg}$ of the corresponding Heck product 9ab (75\% yield). ${ }^{1} \mathrm{H}$ NMR $\left(300 \mathrm{MHz}, \mathrm{CDCl}_{3}\right): 1.39$ (s, 9H), 2.37 (s, 3H), $3.85(\mathrm{~s}, 3 \mathrm{H}), 3.91(\mathrm{~s}, 3 \mathrm{H}), 6.45(\mathrm{~s}, 1 \mathrm{H}), 6.69(\mathrm{~s}, 1 \mathrm{H}), 6.71(\mathrm{~d}, J=2.4 \mathrm{~Hz}, 1 \mathrm{H})$, $6.82(\mathrm{~s}, 1 \mathrm{H}), 7.45(\mathrm{~d}, J=2.4 \mathrm{~Hz}, 1 \mathrm{H}), 7.53(\mathrm{~d}, J=8.4 \mathrm{~Hz}, 2 \mathrm{H}), 8.22(\mathrm{~d}, J=8.4 \mathrm{~Hz}, 2 \mathrm{H}) ;{ }^{13} \mathrm{C} \mathrm{NMR}$ $\left(75 \mathrm{MHz}, \mathrm{CDCl}_{3}\right): \delta 20.7,28.6,52.2,55.5,55.6,60.7,100.1,104.3,113.6,122.8,123.9,124.3$, 127.8, 129.5, 143.1, 147.7, 158.4, 159.3, 161.2, 166.5; LRMS (EI): $453\left(\mathrm{M}^{+}\right)$; HRMS (EI): calcd forC ${ }_{24} \mathrm{H}_{27} \mathrm{~N}_{3} \mathrm{O}_{6}\left(\mathrm{M}^{+}\right)$453.1900, found 453.1888.

\section{Compound 10ab}<smiles>COc1cc2c(C)cn(C(C(=O)NC(C)(C)C)c3ccc([N+](=O)[O-])cc3)c(=O)c2cc1OC</smiles>

$\mathrm{Pd}(\mathrm{OAc})_{2}(5.6 \mathrm{mg}, 0.025 \mathrm{mmol}), \mathrm{Pcy}_{3}(14.0 \mathrm{mg}, 0.05 \mathrm{mmol})$ and 10aa $(290.7 \mathrm{mg}, 0.5 \mathrm{mmol})$ were added to a flame-dried Schlenk tube under nitrogen. DMA (5.0 mL) and $N$-methyldicyclohexylamine $(0.42 \mathrm{~mL}, 2.0 \mathrm{mmol})$ were added under nitrogen. The reaction mixture was stirred at $100{ }^{\circ} \mathrm{C}$ overnight. Solvent was removed under reduced pressure and the residue was purified by flash chromatography $\left(\mathrm{EtOAc} / \mathrm{CH}_{2} \mathrm{Cl}_{2} /\right.$ petroleum ether $\left.=1: 1: 5\right)$ to give $211.4 \mathrm{mg}$ of the corresponding Heck product 10ab (93\% yield). ${ }^{1} \mathrm{H}$ NMR $\left(300 \mathrm{MHz}, \mathrm{CDCl}_{3}\right): 1.39$ (s, 9H), $2.21(\mathrm{~s}, 3 \mathrm{H}), 4.00(\mathrm{~s}, 3 \mathrm{H}), 4.02(\mathrm{~s}, 3 \mathrm{H}), 6.45(\mathrm{~s}, 1 \mathrm{H}), 6.90-6.91(\mathrm{~m}, 3 \mathrm{H}), 7.55(\mathrm{~d}, J=8.4$ $\mathrm{Hz}, 2 \mathrm{H}), 7.79$ (s, $1 \mathrm{H}), 8.22(\mathrm{~d}, J=8.4 \mathrm{~Hz}, 2 \mathrm{H}) ;{ }^{13} \mathrm{C} \mathrm{NMR}\left(75 \mathrm{MHz}, \mathrm{CDCl}_{3}\right): \delta 15.9,28.6,52.3$, 56.1, 56.1, 60.1, 103.6, 108.3, 112.5, 118.9, 124.0, 125.8, 129.5, 132.9, 143.3, 147.7, 149.2, 153.8, 161.4, 166.7; LRMS (EI): $453\left(\mathrm{M}^{+}\right)$; HRMS (EI): calcd forC $\mathrm{C}_{24} \mathrm{H}_{27} \mathrm{~N}_{3} \mathrm{O}_{6}\left(\mathrm{M}^{+}\right) 453.1900$, found 453.1908 .

\section{Compound 1bb}<smiles>COc1cc2c(cc1OC)C(C(=O)NC(C)(C)C)N(C(=O)c1ccc([N+](=O)[O-])cc1)C=C2C</smiles>

$\mathrm{Pd}(\mathrm{OAc})_{2}(5.6 \mathrm{mg}, 0.025 \mathrm{mmol}), \mathrm{Pcy}_{3}(14.0 \mathrm{mg}, 0.05 \mathrm{mmol})$ and $\mathbf{1 b a}(290.7 \mathrm{mg}, 0.5 \mathrm{mmol})$ were added to a flame-dried Schlenk tube under nitrogen. DMA $(5.0 \mathrm{~mL})$ and $N$-methyldicyclohexylamine $(0.42 \mathrm{~mL}, 2.0 \mathrm{mmol})$ were added under nitrogen. The reaction mixture was stirred at $60{ }^{\circ} \mathrm{C}$ overnight. Solvent was removed under reduced pressure and the residue was purified by flash chromatography $\left(\mathrm{EtOAc} / \mathrm{CH}_{2} \mathrm{Cl}_{2} /\right.$ petroleum ether $\left.=1: 1: 3\right)$ to give $203.7 \mathrm{mg}$ of the corresponding Heck product $\mathbf{1 b b}\left(90 \%\right.$ yield). ${ }^{1} \mathrm{H}$ NMR $\left(300 \mathrm{MHz}, \mathrm{CDCl}_{3}\right): 1.24$ 
(s, 9H), $1.99(\mathrm{~s}, 3 \mathrm{H}), 3.94(\mathrm{~s}, 6 \mathrm{H}), 5.91(\mathrm{~s}, 1 \mathrm{H}), 6.03(\mathrm{~s}, 1 \mathrm{H}), 6.18(\mathrm{~s}, 1 \mathrm{H}), 6.83(\mathrm{~s}, 1 \mathrm{H}), 7.76(\mathrm{~d}, J=$ $8.4 \mathrm{~Hz}, 2 \mathrm{H}), 8.31(\mathrm{~d}, J=8.4 \mathrm{~Hz}, 2 \mathrm{H}) ;{ }^{13} \mathrm{C}$ NMR $\left(75 \mathrm{MHz}, \mathrm{CDCl}_{3}\right)$ : $\delta 16.0,28.7,51.6,56.1,56.2$, 58.3, 106.4, 110.4, 117.8, 120.6, 121.4, 123.7, 125.0, 129.8, 140.0, 148.9, 149.0, 149.2, 167.0, 167.6; HRMS (FAB): calcd forC $\mathrm{C}_{24} \mathrm{H}_{28} \mathrm{~N}_{3} \mathrm{O}_{6}\left(\mathrm{M}^{+}\right) 454.1972$, found 454.1976 .

\section{Compound 2bb}<smiles>COc1cc2c(cc1OC)C(C(=O)NC(C)(C)C)N(C(C)=O)C=C2C</smiles>

$\mathrm{Pd}(\mathrm{OAc})_{2}(5.6 \mathrm{mg}, 0.025 \mathrm{mmol}), \mathrm{Pcy}_{3}(14.0 \mathrm{mg}, 0.05 \mathrm{mmol})$ and $2 \mathrm{ba}(237.2 \mathrm{mg}, 0.5 \mathrm{mmol})$ were added to a flame-dried Schlenk tube under nitrogen. DMA (5.0 mL) and $N$-methyldicyclohexylamine $(0.42 \mathrm{~mL}, 2.0 \mathrm{mmol})$ were added under nitrogen. The reaction mixture was stirred at $60{ }^{\circ} \mathrm{C}$ overnight. Solvent was removed under reduced pressure and the residue was purified by flash chromatography $\left(\mathrm{EtOAc} / \mathrm{CH}_{2} \mathrm{Cl}_{2} /\right.$ petroleum ether $\left.=1: 1: 1\right)$ to give $139.0 \mathrm{mg}$ of the corresponding Heck product $\mathbf{2 b b}\left(80 \%\right.$ yield). ${ }^{1} \mathrm{H}$ NMR $\left(300 \mathrm{MHz}, \mathrm{CDCl}_{3}\right): 1.26$ (s, 9H), $2.08(\mathrm{~d}, J=1.2 \mathrm{~Hz}, 3 \mathrm{H}), 2.27(\mathrm{~s}, 1 \mathrm{H}), 3.91(\mathrm{~s}, 3 \mathrm{H}), 3.92(\mathrm{~s}, 3 \mathrm{H}), 5.91(\mathrm{~s}, 1 \mathrm{H}), 5.98(\mathrm{~s}, 1 \mathrm{H})$, $6.79(\mathrm{~d}, J=1.8 \mathrm{~Hz}, 2 \mathrm{H}) ;{ }^{13} \mathrm{C}$ NMR $\left(75 \mathrm{MHz}, \mathrm{CDCl}_{3}\right): \delta 16.2,21.4,28.6,51.34$ 56.0, 56.2, 57.2, 106.1, 110.6, 117.0, 119.9, 121.8, 124.9, 148.5, 148.90 168.4, 169.0; HRMS (FAB): calcd for $\mathrm{C}_{19} \mathrm{H}_{27} \mathrm{~N}_{2} \mathrm{O}_{4}\left(\mathrm{M}^{+}\right)$347.1965, found 347.1971.

\section{Compound 3bb}<smiles>COc1cc2c(cc1OC)C(C(=O)NC1CCCCC1)N(C(=O)c1ccccc1)C=C2C</smiles>

$\mathrm{Pd}(\mathrm{OAc})_{2}(5.6 \mathrm{mg}, 0.025 \mathrm{mmol}), \mathrm{Pcy}_{3}(14.0 \mathrm{mg}, 0.05 \mathrm{mmol})$ and $3 \mathbf{b a}(281.2 \mathrm{mg}, 0.5 \mathrm{mmol})$ were added to a flame-dried Schlenk tube under nitrogen. DMA (5.0 mL) and $N$-methyldicyclohexylamine $(0.42 \mathrm{~mL}, 2.0 \mathrm{mmol})$ were added under nitrogen. The reaction mixture was stirred at $60{ }^{\circ} \mathrm{C}$ overnight. Solvent was removed under reduced pressure and the residue was purified by flash chromatography $\left(\mathrm{EtOAc} / \mathrm{CH}_{2} \mathrm{Cl}_{2} /\right.$ petroleum ether $\left.=1: 1: 3\right)$ to give $195.8 \mathrm{mg}$ of the corresponding Heck product $\mathbf{3 b b}\left(90 \%\right.$ yield). ${ }^{1} \mathrm{H}$ NMR $\left(300 \mathrm{MHz}, \mathrm{CDCl}_{3}\right)$ : 1.20-1.36 (m, 5H), 1.53-1.81 (m, 5H), $1.98(\mathrm{~s}, 3 \mathrm{H}), 3.71(\mathrm{~m}, 1 \mathrm{H}), 3.91(\mathrm{~s}, 3 \mathrm{H}), 3.93(\mathrm{~s}, 3 \mathrm{H}), 6.08$ $(\mathrm{s}, 1 \mathrm{H}), 6.31(\mathrm{~s}, 1 \mathrm{H}), 6.81(\mathrm{~s}, 1 \mathrm{H}), 6.85(\mathrm{~s}, 1 \mathrm{H}), 7.42-7.59(\mathrm{~m}, 5 \mathrm{H}) ;\left(75 \mathrm{MHz}, \mathrm{CDCl}_{3}\right): \quad \delta 16.0$, 24.4, 24.4, 25.4, 32.6, 48.2, 56.0, 57.9, 106.0, 110.6, 116.2, 121.6, 125.2, 128.4, 128.8, 131.1, 134.0, 148.5, 148.9, 168.3, 169.3; HRMS (FAB): calcd for $\mathrm{C}_{26} \mathrm{H}_{31} \mathrm{~N}_{2} \mathrm{O}_{4}\left(\mathrm{M}^{+}\right)$435.2278, found 435.2285 .

\section{Compound 4bb}


<smiles>COc1cc2c(cc1OC)C(C(=O)NC1CCCCC1)N(C(=O)c1ccc([N+](=O)[O-])cc1)C=C2C</smiles>

$\mathrm{Pd}(\mathrm{OAc})_{2}(5.6 \mathrm{mg}, 0.025 \mathrm{mmol}), \mathrm{Pcy}_{3}(14.0 \mathrm{mg}, 0.05 \mathrm{mmol})$ and $4 \mathbf{b a}(303.7 \mathrm{mg}, 0.5 \mathrm{mmol})$ were added to a flame-dried Schlenk tube under nitrogen. DMA $(5.0 \mathrm{~mL})$ and $N$-methyldicyclohexylamine $(0.42 \mathrm{~mL}, 2.0 \mathrm{mmol})$ were added under nitrogen. The reaction mixture was stirred at $60{ }^{\circ} \mathrm{C}$ overnight. Solvent was removed under reduced pressure and the residue was purified by flash chromatography $\left(\mathrm{EtOAc} / \mathrm{CH}_{2} \mathrm{Cl}_{2} /\right.$ petroleum ether $\left.=1: 1: 3\right)$ to give $218.5 \mathrm{mg}$ of the corresponding Heck product $4 \mathbf{b b}\left(91 \%\right.$ yield). ${ }^{1} \mathrm{H}$ NMR $\left(300 \mathrm{MHz}, \mathrm{CDCl}_{3}\right)$ : 1.08-1.25 (m, 5H), 1.59-1.86 (m, 5H), $1.98(\mathrm{~s}, 3 \mathrm{H}), 3.71(\mathrm{~m}, 1 \mathrm{H}), 3.95(\mathrm{~s}, 6 \mathrm{H}), 6.00(\mathrm{~d}, J=7.2 \mathrm{~Hz}$, $1 \mathrm{H}), 6.11(\mathrm{~s}, 1 \mathrm{H}), 6.18(\mathrm{~s}, 1 \mathrm{H}), 6.82(\mathrm{~s}, 1 \mathrm{H}), 6.91(\mathrm{~s}, 1 \mathrm{H}), 7.75(\mathrm{~d}, J=8.4 \mathrm{~Hz}, 2 \mathrm{H}), 8.32(\mathrm{~d}, J=8.4$ $\mathrm{Hz}, 2 \mathrm{H}) ;\left(75 \mathrm{MHz}, \mathrm{CDCl}_{3}\right): \delta 16.0,24.4,25.5,32.7,56.0,56.1,57.8,106.3,110.4,117.6,120.6$, 121.1, 123.7, 125.0, 129.6, 140.1, 148.8, 148.9, 149.2, 167.0, 167.5; HRMS (FAB): calcd for $\mathrm{C}_{26} \mathrm{H}_{30} \mathrm{~N}_{3} \mathrm{O}_{6}\left(\mathrm{M}^{+}\right)$480.2129, found 480.2125 .

\section{Compound 5bb}<smiles>COc1ccc(C(=O)N2C=C(C)c3cc(OC)c(OC)cc3C2C(=O)NC2CCCCC2)cc1</smiles>

$\mathrm{Pd}(\mathrm{OAc})_{2}(5.6 \mathrm{mg}, 0.025 \mathrm{mmol}), \mathrm{Pcy}_{3}(14.0 \mathrm{mg}, 0.05 \mathrm{mmol})$ and $5 \mathbf{b a}(296.2 \mathrm{mg}, 0.5 \mathrm{mmol})$ were added to a flame-dried Schlenk tube under nitrogen. DMA (5.0 mL) and $N$-methyldicyclohexylamine $(0.42 \mathrm{~mL}, 2.0 \mathrm{mmol})$ were added under nitrogen. The reaction mixture was stirred at $60{ }^{\circ} \mathrm{C}$ overnight. Solvent was removed under reduced pressure and the residue was purified by flash chromatography $\left(\mathrm{EtOAc} / \mathrm{CH}_{2} \mathrm{Cl}_{2} /\right.$ petroleum ether $\left.=1: 1: 3\right)$ to give $213.7 \mathrm{mg}$ of the corresponding Heck product $\mathbf{5 b b}\left(92 \%\right.$ yield). ${ }^{1} \mathrm{H}$ NMR $\left(300 \mathrm{MHz}, \mathrm{CDCl}_{3}\right)$ : 1.14-1.35 (m, 5H), 1.53-1.85 (m, 5H), $2.01(\mathrm{~s}, 3 \mathrm{H}), 3.71(\mathrm{~m}, 1 \mathrm{H}), 3.87(\mathrm{~s}, 3 \mathrm{H}), 3.90(\mathrm{~s}, 3 \mathrm{H}), 3.93$ (s, 3H), $6.00(\mathrm{~s}, 1 \mathrm{H}), 6.39(\mathrm{~s}, 1 \mathrm{H}), 6.47(\mathrm{~s}, 1 \mathrm{H}), 6.39(\mathrm{~s}, 1 \mathrm{H}), 6.47(\mathrm{~s}, 1 \mathrm{H}), 6.82(\mathrm{~d}, J=8.7 \mathrm{~Hz}, 2 \mathrm{H})$, 7.58 (d, $J=8.7 \mathrm{~Hz}, 2 \mathrm{H}) ;\left(75 \mathrm{MHz}, \mathrm{CDCl}_{3}\right): \delta 15.9,24.4,25.4,32.6,48.0,55.3,55.9,56.0,58.2$, 105.9, 110.6, 113.6, 121.9, 125.2, 125.9, 129.4, 131.0, 148.4, 148.8, 161.8, 168.5, 158.8; HRMS (FAB): calcd for $\mathrm{C}_{27} \mathrm{H}_{33} \mathrm{~N}_{2} \mathrm{O}_{5}\left(\mathrm{M}^{+}\right) 465.2384$, found 465.2394 .

\section{Compound 6bb}


<smiles>CC(=O)N1C=C(C)c2ccccc2C1C(=O)NC(C)(C)C</smiles>

$\mathrm{Pd}(\mathrm{OAc})_{2}(5.6 \mathrm{mg}, 0.025 \mathrm{mmol}), \mathrm{Pcy}_{3}(14.0 \mathrm{mg}, 0.05 \mathrm{mmol})$ and $6 \mathbf{b a}(183.6 \mathrm{mg}, 0.5 \mathrm{mmol})$ were added to a flame-dried Schlenk tube under nitrogen. DMA (5.0 mL) and $N$-methyldicyclohexylamine $(0.42 \mathrm{~mL}, 2.0 \mathrm{mmol})$ were added under nitrogen. The reaction mixture was stirred at $60{ }^{\circ} \mathrm{C}$ overnight. Solvent was removed under reduced pressure and the residue was purified by flash chromatography $\left(\mathrm{EtOAc} / \mathrm{CH}_{2} \mathrm{Cl}_{2} /\right.$ petroleum ether $\left.=1: 1: 3\right)$ to give $122.0 \mathrm{mg}$ of the corresponding Heck product $\mathbf{6 b b}\left(85 \%\right.$ yield). ${ }^{1} \mathrm{H}$ NMR $\left(300 \mathrm{MHz}, \mathrm{CDCl}_{3}\right): 1.24$ (s, 9H), 2.29 (s, 3H), $5.79(\mathrm{~s}, 1 \mathrm{H}), 6.05(\mathrm{~s}, 1 \mathrm{H}), 7.21-7.35(\mathrm{~m}, 4 \mathrm{H}) ;{ }^{13} \mathrm{C} \mathrm{NMR}\left(75 \mathrm{MHz}, \mathrm{CDCl}_{3}\right)$ : $\delta$ 16.0, 21.3, 28.4, 28.6, 51.4, 57.5, 116.6, 121.9, 122.5, 127.0, 127.6, 128.5, 129.2, 131.8, 168.1, 168.9; HRMS (FAB): calcd for $\mathrm{C}_{17} \mathrm{H}_{23} \mathrm{~N}_{2} \mathrm{O}_{2}\left(\mathrm{M}^{+}\right)$287.1754, found 287.1752 .

\section{Compound 7bb}<smiles>COc1ccc(C(=O)N2C=C(C)c3ccccc3C2C(=O)NC2CCCCC2)cc1</smiles>

$\mathrm{Pd}(\mathrm{OAc})_{2}(5.6 \mathrm{mg}, 0.025 \mathrm{mmol}), \mathrm{Pcy}_{3}(14.0 \mathrm{mg}, 0.05 \mathrm{mmol})$ and $7 \mathrm{ba}(242.7 \mathrm{mg}, 0.5 \mathrm{mmol})$ were added to a flame-dried Schlenk tube under nitrogen. DMA (5.0 mL) and $N$-methyldicyclohexylamine $(0.42 \mathrm{~mL}, 2.0 \mathrm{mmol})$ were added under nitrogen. The reaction mixture was stirred at $60{ }^{\circ} \mathrm{C}$ overnight. Solvent was removed under reduced pressure and the residue was purified by flash chromatography $\left(\mathrm{EtOAc} / \mathrm{CH}_{2} \mathrm{Cl}_{2} /\right.$ petroleum ether $\left.=1: 1: 5\right)$ to give $152.1 \mathrm{mg}$ of the corresponding Heck product $7 \mathbf{b b}\left(75 \%\right.$ yield). ${ }^{1} \mathrm{H}$ NMR $\left(300 \mathrm{MHz}, \mathrm{CDCl}_{3}\right)$ : 1.10-1.37 (m, 5H), 1.51-2.01 (m, 5H), $2.17(\mathrm{~s}, 3 \mathrm{H}), 3.70(\mathrm{~m}, 1 \mathrm{H}), 3.87(\mathrm{~s}, 3 \mathrm{H}), 6.05(\mathrm{~s}, 1 \mathrm{H}), 6.31$ (s, $1 \mathrm{H}), 6.48(\mathrm{~s}, 1 \mathrm{H}), 6.94(\mathrm{~d}, J=8.7 \mathrm{~Hz}, 2 \mathrm{H}), 7.28-7.41(\mathrm{~m}, 4 \mathrm{H}), 7.58(\mathrm{~d}, J=8.7 \mathrm{~Hz}, 2 \mathrm{H}) ;(75 \mathrm{MHz}$, $\left.\mathrm{CDCl}_{3}\right): \quad \delta 15.7,24.4,25.4,32.6,32.6,48.2,55.4,113.7,122.3,125.9,127.5,128.6,127.5,128.6$, 129.1, 131.1, 132.3, 161.9, 168.2; HRMS (FAB): calcd for $\mathrm{C}_{25} \mathrm{H}_{29} \mathrm{~N}_{2} \mathrm{O}_{3}\left(\mathrm{M}^{+}\right) 405.2173$, found 405.2172 .

\section{Compound 8bb}<smiles>COc1ccc(C(=O)N2C=C(C)c3ccccc3C2C(=O)NC(C)(C)C)cc1</smiles>

$\mathrm{Pd}(\mathrm{OAc})_{2}(5.6 \mathrm{mg}, 0.025 \mathrm{mmol}), \mathrm{Pcy}_{3}(14.0 \mathrm{mg}, 0.05 \mathrm{mmol})$ and $8 \mathbf{b a}(229.7 \mathrm{mg}, 0.5 \mathrm{mmol})$ were added to a flame-dried Schlenk tube under nitrogen. DMA $(5.0 \mathrm{~mL})$ and 
$N$-methyldicyclohexylamine $(0.42 \mathrm{~mL}, 2.0 \mathrm{mmol})$ were added under nitrogen. The reaction mixture was stirred at $60{ }^{\circ} \mathrm{C}$ overnight. Solvent was removed under reduced pressure and the residue was purified by flash chromatography $\left(\mathrm{EtOAc} / \mathrm{CH}_{2} \mathrm{Cl}_{2} /\right.$ petroleum ether $\left.=1: 1: 3\right)$ to give $164.2 \mathrm{mg}$ of the corresponding Heck product $8 \mathbf{b b}\left(87 \%\right.$ yield). ${ }^{1} \mathrm{H}$ NMR $\left(300 \mathrm{MHz}, \mathrm{CDCl}_{3}\right): 1.27$ (s, 9H), $2.02(\mathrm{~s}, 3 \mathrm{H}), 3.87(\mathrm{~s}, 3 \mathrm{H}), 6.00(\mathrm{~s}, 1 \mathrm{H}), 6.32(\mathrm{~s}, 1 \mathrm{H}), 6.48(\mathrm{~s}, 1 \mathrm{H}), 6.94(\mathrm{~d}, J=9.0 \mathrm{~Hz}, 2 \mathrm{H})$, 7.24-7.41 (m, 4H), $7.59(\mathrm{~d}, \quad J=9.0 \mathrm{~Hz}, 2 \mathrm{H}) ;{ }^{13} \mathrm{C} \mathrm{NMR}\left(75 \mathrm{MHz}, \mathrm{CDCl}_{3}\right): \quad \delta 15.7,28.6,51.3$, 58.9, 113.6, 113.7, 122.3, 123.6, 125.8, 127.1, 127.4, 128.4, 129.2, 129.5, 131.1, 132.1, 161.8, 168.3; HRMS (FAB): calcd for $\mathrm{C}_{23} \mathrm{H}_{27} \mathrm{~N}_{2} \mathrm{O}_{3}\left(\mathrm{M}^{+}\right)$379.2016, found 379.2011. 